\title{
Theory of the Josephson effect in unconventional superconducting junctions with diffusive barriers
}

\author{
T. Yokoyama, ${ }^{1}$ Y. Tanaka, ${ }^{1}$ and A. A. Golubov ${ }^{2}$ \\ ${ }^{1}$ Department of Applied Physics, Nagoya University, Nagoya, 464-8603, Japan \\ and CREST, Japan Science and Technology Corporation (JST) Nagoya, 464-8603, Japan \\ ${ }^{2}$ Faculty of Science and Technology, University of Twente, 7500 AE, Enschede, The Netherlands \\ (Received 6 November 2006; revised manuscript received 9 January 2007; published 30 March 2007)
}

\begin{abstract}
We study theoretically the Josephson effect in junctions based on unconventional superconductors with diffusive barriers, using the quasiclassical Green's function formalism. Generalized boundary conditions at junction interfaces applicable to unconventional superconductors are derived by calculating a matrix current within the circuit transport theory. Applying these boundary conditions, we have calculated the Josephson current in structures with various pairing symmetries. A number of predictions are made: (a) nonmonotonic temperature dependence in $d$-wave superconductor/diffusive normal metal/ $d$-wave superconductor (D/DN/D) junctions, (b) anomalous current-phase relations in $p$-wave superconductor/diffusive normal metal $/ p$-wave superconductor (P/DN/P) junctions, (c) second harmonics in D/DN/D and P/DN/P junctions, (d) a double-peak structure of the critical current in $\mathrm{D} / \mathrm{DF} / \mathrm{D}$ junctions, and (e) enhanced Josephson current by the exchange field in S/DF/P junctions. We have also investigated peculiarities of the Josephson coupling in D/DF/D, P/DF/P, and $\mathrm{S} / \mathrm{DF} / \mathrm{P}$ junctions. An oscillatory behavior of the supercurrent and the second harmonics in the current-phase relation is studied as a function of the length of the diffusive ferromagnet.
\end{abstract}

DOI: 10.1103/PhysRevB.75.094514

PACS number(s): 74.20.Rp, 74.50.+r, 74.70.Kn

\section{INTRODUCTION}

The Josephson effect ${ }^{1}$ has been studied in various types of junctions ${ }^{2-4}$ motivated by fundamental interest and potential applications for future technology. In superconductor/ diffusive normal metal/superconductor (S/DN/S) junctions the critical current increases monotonically with decreasing temperature $^{3-6}$ because the proximity effect is enhanced at low temperatures. Superconducting junctions with ferromagnetic interlayers have shown rich physics due to the interplay of the proximity effect and the exchange field. ${ }^{7,8}$ When the $\mathrm{DN}$ is replaced by a diffusive ferromagnet (DF), it was predicted that $\pi$ junctions can be realized. ${ }^{9-16}$ The physical reason for a $\pi$ state is nonzero momentum of induced Cooper pairs in the ferromagnet, ${ }^{12}$ similar to the so-called FuldeFerrel-Larkin-Ovchinnikov state ${ }^{17,18}$ in a magnetic superconductor. SFS $\pi$ junctions have been realized experimentally by several groups. ${ }^{19-29}$

In $d$-wave superconductor junctions, one of the most remarkable phenomena is the formation of midgap Andreev resonant states (MARSs) at interfaces. ${ }^{30}$ The MARSs stem from a sign change of pair potentials of $d$-wave superconductors. $^{31}$ In $d$-wave superconductor/insulator/ $d$-wave superconductor junctions, $\pi$ junctions emerge due to the formation of the MARSs. ${ }^{32,33}$ In order to clarify the role of the proximity effect and MARSs, Tanaka et al. have extended circuit theory ${ }^{34}$ to junctions with unconventional superconductors. ${ }^{35-38}$ The conservation of matrix current enables one to apply the generalized Kirchhoff rules to unconventional superconducting junctions and to derive the boundary conditions for the Usadel equation ${ }^{39}$ widely used in diffusive superconducting junctions. Application of this theory to $\mathrm{DN} / d$-wave superconductor (DN/D) junctions has shown that the formation of MARSs strongly competes with the proximity effect in DNs. ${ }^{35,36}$ It was also demonstrated that the formation of MARSs coexists with the proximity effect in $\mathrm{DN} / p$-wave superconductor (DN/P) junctions, which produces a giant zero-bias conductance peak. ${ }^{37}$

Recently this theory has been extended to diffusive Josephson junctions with unconventional superconductors. ${ }^{40}$ It is clarified that a nonmonotonic temperature dependence of the critical current appears in D/DN/D junctions due to the competition between the proximity effect and the formation of MARSs. Concerning unconventional superconducting junctions with clean ferromagnets, the Josephson effect is studied in Ref. 41. However, the Josephson effect in unconventional superconducting junctions with diffusive ferromagnets has not yet been studied, although the proximity effect in these junctions was studied recently. ${ }^{42}$ Moreover, detailed derivation of the boundary conditions is not given and only D/DN/D junctions are considered in Ref. 40. Since the proximity effect and MARSs strongly influence the density of states, ${ }^{35-37}$ they should also crucially influence the Josephson effect in diffusive unconventional superconducting junctions.

The purpose of the present paper is to study the Josephson effect in various types of conventional and unconventional superconducting junctions with DN or DF interlayers. We first provide the technical details of the derivation in Ref. 40. Then, solving the Usadel equations, we apply the general approach to study the influence of the exchange field, the proximity effect, and the formation of MARSs on the Josephson current simultaneously. A number of peculiarities in the Josephson current are found depending on the pairing symmetry: a nonmonotonic temperature dependence in $\mathrm{D} / \mathrm{DN} / \mathrm{D}$ junctions, anomalous current-phase relations in $\mathrm{P} / \mathrm{DN} / \mathrm{P}$ junctions, second harmonics in the current-phase relation and their half-periodic oscillations as a function of the length of the DF in D/DN/D and P/DN/P junctions, transitions to a $\pi$ state in $\mathrm{D} / \mathrm{DF} / \mathrm{D}, \mathrm{P} / \mathrm{DF} / \mathrm{P}$, and S/DF/P junctions, a double-peak structure in the temperature dependence of the 
critical current in D/DF/D junctions, and enhancement of Josephson current by the exchange field in S/DF/P junctions.

\section{FORMULATION}

We consider a junction consisting of unconventional superconductors (USCs) connected by a quasi-one-dimensional $\mathrm{DN}$ (or DF) with a resistance $R_{d}$ and a length $L$ much larger than the mean free path. The DN/USC interface located at $x=0$ has the resistance $R_{b}^{\prime}$, while the DN/USC interface located at $x=L$ has the resistance $R_{b}$. We model infinitely narrow insulating barriers by the delta function $U(x)=H \delta(x$ $-L)+H^{\prime} \delta(x)$. The resulting transparencies of the junctions $T_{m}$ and $T_{m}^{\prime}$ are given by $T_{m}=4 \cos ^{2} \phi /\left(4 \cos ^{2} \phi+Z^{2}\right)$ and $T_{m}^{\prime}=4 \cos ^{2} \phi /\left(4 \cos ^{2} \phi+Z^{\prime 2}\right)$, where $m$ is a channel index, $Z=2 H / v_{F}$ and $Z^{\prime}=2 H^{\prime} / v_{F}$ are dimensionless constants, $\phi$ is the injection angle measured from the interface normal to the junction, and $v_{F}$ is the Fermi velocity.

In order to study the Josephson effect in diffusive USC junctions, we first concentrate on the quasiclassical KeldyshNambu Green's function in a DN defined by $\check{G}_{N}(x)$. Its retarded part $\hat{R}_{N}(x)$ can be expressed as

$$
\hat{R}_{N}(x)=\cos \psi \sin \theta \hat{\tau}_{1}+\sin \psi \sin \theta \hat{\tau}_{2}+\cos \theta \hat{\tau}_{3},
$$

with Pauli matrices in the electron-hole space $\hat{\tau}_{1}, \hat{\tau}_{2}$, and $\hat{\tau}_{3}$. Since $\hat{R}_{N}(x)$ obeys the Usadel equation, the following equations are satisfied:

$$
\begin{gathered}
D\left[\frac{\partial^{2}}{\partial x^{2}} \theta-\left(\frac{\partial \psi}{\partial x}\right)^{2} \cos \theta \sin \theta\right]+2 i[\varepsilon+(-) h] \sin \theta=0, \\
\frac{\partial}{\partial x}\left[\sin ^{2} \theta\left(\frac{\partial \psi}{\partial x}\right)\right]=0
\end{gathered}
$$

for majority (minority) spin with the diffusion constant $D$ and exchange field $h$. The boundary condition of $\check{G}_{N}(x)$ at the DN/USC interface is given by ${ }^{37,40}$

$$
\begin{gathered}
\left.\frac{L}{R_{d}}\left(\check{G}_{N}(x) \frac{\partial \check{G}_{N}(x)}{\partial x}\right)\right|_{x=L_{-}}=-\frac{\langle\check{I}(\phi)\rangle}{R_{b}} \\
\check{I}(\phi)=2\left[\check{G}_{1}, \check{B}\right], \\
\check{B}=\left(-T_{1}\left[\check{G}_{1}, \check{H}_{-}^{-1}\right]+\check{H}_{-}^{-1} \check{H}_{+}-T_{1}^{2} \check{G}_{1} \check{H}_{-}^{-1} \check{H}_{+} \check{G}_{1}\right)^{-1}\left[T_{1}\left(1-\check{H}_{-}^{-1}\right)\right. \\
\left.+T_{1}^{2} \check{G}_{1} \check{H}_{-}^{-1} \check{H}_{+}\right]
\end{gathered}
$$

with $\check{G}_{1}=\check{G}_{N}\left(x=L_{-}\right), \check{H}_{ \pm}=\left(\check{G}_{2+} \pm \check{G}_{2-}\right) / 2$, and $T_{1}=T /(2-T$ $+2 \sqrt{1-T}$ ), where $\check{G}_{2 \pm}$ is the asymptotic Green's function in USCs as defined in our previous papers. ${ }^{37}\langle\rangle$ means the average over the various angles of injected particles at the interface. The retarded components of $\check{G}_{1}$ and $\check{G}_{2 \pm}$ are given by $\hat{R}_{1,2 \pm}$ where $\hat{R}_{2 \pm}$ is expressed by $\hat{R}_{2 \pm}=g_{ \pm} \hat{\tau}_{3}+f_{ \pm} \hat{\tau}_{2}$ with $g_{ \pm}$ $=\varepsilon / \sqrt{\varepsilon^{2}-\Delta_{ \pm}^{2}}$ and $f_{ \pm}=\Delta_{ \pm} / \sqrt{\Delta_{ \pm}^{2}-\varepsilon^{2}}$, where $\varepsilon$ denotes the qua- siparticle energy measured from the Fermi energy. $\Delta_{+}\left(\Delta_{-}\right)$is the effective pair potential experienced by quasiparticles with an injection angle $\phi(\pi-\phi)$. We also denote by $\hat{R}_{p}, \hat{R}_{m}$, and $\hat{I}_{R}$ the retarded parts of $\check{H}_{+}, \check{H}_{-}$, and $\check{I}$.

Now we discuss the boundary condition of the retarded part of the Keldysh-Nambu Green's function at the DN/USC interface. The left side of the boundary condition of Eq. (4) can be expressed as

$$
\begin{aligned}
\frac{L}{R_{d}} \hat{R}_{N}(x) & \left.\frac{\partial}{\partial x} \hat{R}_{N}(x)\right|_{x=L} \\
= & \frac{L i}{R_{d}}\left[\left(-\frac{\partial \theta}{\theta x} \sin \psi-\frac{\partial \psi}{\theta x} \sin \theta \cos \theta \cos \psi\right) \hat{\tau}_{1}\right. \\
& +\left(\frac{\partial \theta}{\theta x} \cos \psi-\frac{\partial \psi}{\theta x} \sin \theta \cos \theta \sin \psi\right) \hat{\tau}_{2} \\
& \left.+\frac{\partial \psi}{\theta x} \sin ^{2} \theta \hat{\tau}_{3}\right] .
\end{aligned}
$$

In the right side of Eq. (4), $\hat{I}_{R}$ can be expressed by using several spectral vectors:

$$
\begin{aligned}
\hat{I}_{R}= & 4 i T_{1}\left(d_{R} \cdot d_{R}\right)^{-1}\left(-\frac{1}{2}\left(1+T_{1}^{2}\right)\left(s_{2+}-s_{2-}\right)^{2}\left[s_{1} \times\left(s_{2+}\right.\right.\right. \\
& \left.\left.+s_{2-}\right)\right] \cdot \hat{\boldsymbol{\tau}}+2 T_{1} s_{1} \cdot\left(s_{2+} \times s_{2-}\right)\left[s_{1} \times\left(s_{2+} \times s_{2-}\right)\right] \cdot \hat{\boldsymbol{\tau}} \\
& +2 T_{1} s_{1} \cdot\left(s_{2+}-s_{2-}\right)\left[s_{1} \times\left(s_{2+}-s_{2-}\right)\right] \cdot \hat{\tau}-i\left(1+T_{1}^{2}\right)(1 \\
& \left.-s_{2+} \cdot \boldsymbol{s}_{2-}\right)\left[s_{1} \times\left(s_{2+} \times s_{2-}\right)\right] \cdot \hat{\boldsymbol{\tau}}+2 i T_{1}\left(1-s_{2+} \cdot \boldsymbol{s}_{2-}\right) \\
& \left.\times\left[s_{1} \cdot\left(\boldsymbol{s}_{2+}-\boldsymbol{s}_{2-}\right) \boldsymbol{s}_{1}-\left(\boldsymbol{s}_{2+}-\boldsymbol{s}_{2-}\right)\right] \cdot \hat{\boldsymbol{\tau}}\right) \\
& \boldsymbol{d}_{R}=\left(1+T_{1}^{2}\right)\left(\boldsymbol{s}_{2+} \times \boldsymbol{s}_{2-}\right)-2 T_{1} \boldsymbol{s}_{1} \times\left(\boldsymbol{s}_{2+}-\boldsymbol{s}_{2-}\right) \\
& -2 T_{1}^{2} \boldsymbol{s}_{1} \cdot\left(\boldsymbol{s}_{2+} \times \boldsymbol{s}_{2-}\right) \boldsymbol{s}_{1}
\end{aligned}
$$

with $\hat{R}_{1}=s_{1} \cdot \hat{\boldsymbol{\tau}}$ and $\hat{R}_{2 \pm}=\boldsymbol{s}_{2 \pm} \cdot \hat{\boldsymbol{\tau}}^{37}$

The spectral vectors $\boldsymbol{s}_{1}$ and $\boldsymbol{s}_{2 \pm}$ are given by

$$
\boldsymbol{s}_{1}=\left(\begin{array}{c}
\sin \theta \cos \psi \\
\sin \theta \sin \psi \\
\cos \theta
\end{array}\right), \quad \boldsymbol{s}_{2 \pm}=\left(\begin{array}{c}
f_{ \pm} \cos \Psi \\
f_{ \pm} \sin \Psi \\
g_{ \pm}
\end{array}\right)
$$

where $\Psi$ denotes the phase of the USC. Then $\hat{I}_{R}$ is reduced to

$$
\begin{aligned}
\hat{I}_{R}= & 2 i T\left\{(2-T)+T\left[\bar{f}_{S} \sin \theta \cos (\psi-\Psi)+g_{S} \cos \theta\right]\right. \\
& \left.-T f_{S} \sin \theta \sin (\psi-\Psi)\right\}^{-1}\left\{\left(-g_{S} \sin \theta \sin \psi\right.\right. \\
& \left.+\bar{f}_{S} \cos \theta \sin \Psi-f_{S} \cos \theta \cos \Psi\right) \hat{\tau}_{1}+\left(-\bar{f}_{S} \cos \theta \cos \Psi\right. \\
& \left.+g_{S} \sin \theta \cos \psi-f_{S} \cos \theta \sin \Psi\right) \hat{\tau}_{2}+\left[\bar{f}_{S} \sin \theta \sin (\psi\right. \\
& \left.\left.-\Psi)+f_{S} \sin \theta \cos (\psi-\Psi)\right] \hat{\tau}_{3}\right\},
\end{aligned}
$$

and hence we find the following form of the matrix current: 
$\left\langle\hat{I}_{R}\right\rangle$

$$
\begin{gathered}
=i\left(\begin{array}{c}
-I_{1} \sin \theta \sin \psi+I_{2} \cos \theta \sin \Psi-I_{3} \cos \theta \cos \Psi \\
-I_{2} \cos \theta \cos \Psi+I_{1} \sin \theta \cos \psi-I_{3} \cos \theta \sin \Psi \\
I_{2} \sin \theta \sin (\psi-\Psi)+I_{3} \sin \theta \cos (\psi-\Psi)
\end{array}\right) \cdot \hat{\boldsymbol{\tau}}, \\
I_{1}=\left\langle\frac{2 T_{m} g_{S}}{A}\right\rangle, \quad I_{2}=\left\langle\frac{2 T_{m} \bar{f}_{S}}{A}\right\rangle, \quad I_{3}=\left\langle\frac{2 T_{m} f_{S}}{A}\right\rangle, \\
A=\left(2-T_{m}\right)+T_{m}\left[\bar{f}_{S} \sin \theta \cos (\psi-\Psi)+g_{S} \cos \theta\right] \\
-T_{m} f_{S} \sin \theta \sin (\psi-\Psi), \\
g_{S}=\frac{g_{+}+g_{-}}{1+f_{+} f_{-}+g_{+} g_{-}}, \quad \bar{f}_{S}=\frac{f_{+}+f_{-}}{1+f_{+} f_{-}+g_{+} g_{-}}, \\
f_{S}=\frac{i\left(f_{+} g_{-}-f_{-} g_{+}\right)}{1+f_{+} f_{-}+g_{+} g_{-}} .
\end{gathered}
$$

Finally the boundary conditions are given by

$$
\begin{gathered}
\frac{L R_{b}}{R_{d}} \frac{\partial}{\partial x} \theta=-I_{1} \sin \theta+I_{2} \cos \theta \cos (\psi-\Psi) \\
-I_{3} \cos \theta \sin (\psi-\Psi), \\
\frac{L R_{b}}{R_{d}} \sin \theta \frac{\partial}{\partial x} \psi=-I_{2} \sin (\psi-\Psi)-I_{3} \cos (\psi-\Psi) .
\end{gathered}
$$

For the calculation of the thermodynamical quantities, we use the Matsubara representation: $\varepsilon \rightarrow i \omega$. We parametrize the quasiclassical Green's functions $G$ and $F$ using the function $\Phi$ :

$$
\begin{gathered}
G_{\omega}=\frac{\omega}{\sqrt{\omega^{2}+\Phi_{\omega} \Phi_{-\omega}^{*}}}=\cos \theta, \\
F_{\omega}=\frac{\Phi_{\omega}}{\sqrt{\omega^{2}+\Phi_{\omega} \Phi_{-\omega}^{*}}}=\frac{\Phi_{\omega}}{\omega} G_{\omega}=\sin \theta e^{-i \psi}, \\
F_{-\omega}^{*}=\frac{\Phi_{-\omega}^{*}}{\sqrt{\omega^{2}+\Phi_{\omega} \Phi_{-\omega}^{*}}}=\frac{\Phi_{-\omega}^{*}}{\omega} G_{\omega}=\sin \theta e^{i \psi}
\end{gathered}
$$

with Matsubara frequency $\omega$. Then the Usadel equation reads $^{39}$

$$
\xi^{2} \frac{\pi T_{C}}{G_{\omega}} \frac{\partial}{\partial x}\left(G_{\omega}^{2} \frac{\partial}{\partial x} \Phi_{\omega}\right)-[\omega-(+) i h] \Phi_{\omega}=0
$$

for majority (minority) spin with $\xi=\sqrt{D / 2 \pi T_{C}}$ and critical temperature $T_{C}$. The following relations are satisfied:

$$
\begin{aligned}
& \sin \theta \cos \psi=\frac{G_{\omega}}{2 \omega}\left(\Phi_{\omega}+\Phi_{-\omega}^{*}\right), \\
& \sin \theta \sin \psi=\frac{i G_{\omega}}{2 \omega}\left(\Phi_{\omega}-\Phi_{-\omega}^{*}\right) .
\end{aligned}
$$

Then the boundary condition is expressed as

$$
\begin{gathered}
\frac{G_{\omega}}{\omega} \frac{\partial}{\partial x} \Phi_{\omega}=\frac{R_{d}}{R_{b} L}\left(-\frac{\Phi_{\omega}}{\omega} I_{1}+e^{-i \Psi}\left(I_{2}+i I_{3}\right)\right), \\
I_{1}=\left\langle\frac{2 T_{m} g_{S}}{A}\right\rangle, \quad I_{2}=\left\langle\frac{2 T_{m} \bar{f}_{S}}{A}\right\rangle, \quad I_{3}=\left\langle\frac{2 T_{m} f_{S}}{A}\right\rangle, \\
A=2-T_{m}+T_{m}\left[g_{S} G_{\omega}+\bar{f}_{S}(B \cos \Psi+C \sin \Psi)\right. \\
\left.-f_{S}(C \cos \Psi-B \sin \Psi)\right], \\
B=\frac{G_{\omega}}{2 \omega}\left(\Phi_{\omega}+\Phi_{-\omega}^{*}\right), \quad C=\frac{i G_{\omega}}{2 \omega}\left(\Phi_{\omega}-\Phi_{-\omega}^{*}\right),
\end{gathered}
$$

at $x=L$.

This boundary condition is quite general since with a proper choice of $\Delta_{ \pm}$, it is applicable to any unconventional superconductor with $S_{z}=0$ in a time reversal symmetry conserving state. Here, $S_{z}$ denotes the $z$ component of the total spin of a Cooper pair. For $s-, d$-, and $p$-wave superconductors we choose $\Delta_{ \pm}=\Delta(T), \quad \Delta(T) \cos (2 \phi \mp 2 \alpha)$, and $\Delta(T) \cos (\phi \mp \alpha)$, respectively.

In the following we will calculate the Josephson current using this boundary condition at $x=0$ and $L$, where $\varphi$ is the external phase difference across the junctions, and $\alpha$ and $\beta$ denote the angles between the normal to the interface and the crystal axes of the USCs for $x \leqslant 0$ and $x \geqslant L$, respectively. It is important to note that the solution of the Usadel equation is invariant under the transformation $\alpha \rightarrow-\alpha$ or $\beta \rightarrow-\beta$. This is made clear by replacing $\phi$ with $-\phi$ in the angular averaging.

The Josephson current can be expressed using $\Phi_{\omega}$ and $\Phi_{-\omega}^{*}{ }^{*}$ Below $R, T$, and $I_{C}$ denote $R_{d}+R_{b}+R_{b}^{\prime}$, the temperature, and the critical current, respectively, and we consider symmetric barriers with $R_{b}=R_{b}^{\prime}$ and $Z=Z^{\prime}$ for simplicity.

\section{RESULTS}

\section{A. Junctions with DNs}

Let us first focus on the junctions with DNs. Figure 1 shows the current-phase relation for $T / T_{C}=0.1, R_{d} / R_{b}=0.1$, and $E_{T h} / \Delta(0)=0.2$ in (a) $s$-wave, (b) $d$-wave, and (c) $p$-wave superconducting junctions with $(\alpha, \beta)=(0,0)$. In $s$-wave junctions, the $I R$ product is suppressed with the increase of $Z$ because the proximity effect is suppressed. In $d$-wave junctions, the proximity effect and hence $I R$ are enhanced with the increase of $Z$ because of the cancellation of the positive and negative parts of the pair potential in the angular averaging. As $Z$ increases, the contribution from the positive part exceeds that from the negative part and hence the cancellation becomes weak. ${ }^{40}$ In $p$-wave junctions, the $I R$ product is strongly enhanced with the increase of $Z$ because of the formation of resonant states. It is known that the proximity effect and MARSs can coexist. ${ }^{37}$ At $(\alpha, \beta)=(0,0)$, the proximity effect is mostly enhanced. As $Z$ increases, the contribution of the MARSs becomes remarkable and hence the proximity effect gets strongly enhanced. ${ }^{37}$ Consequently its magnitude is an order of magnitude larger than that in 


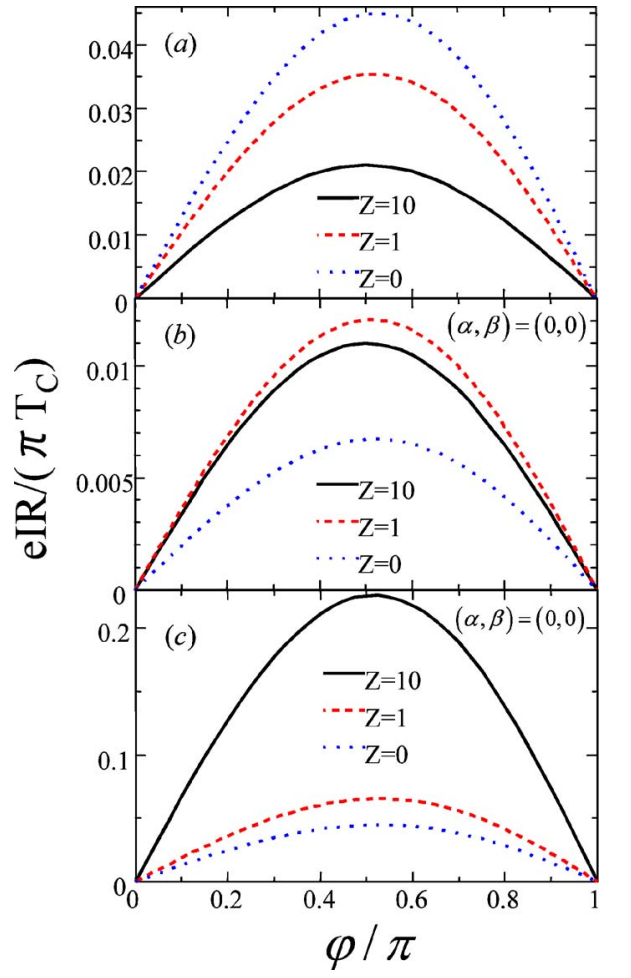

FIG. 1. (Color online) Current-phase relation for $T / T_{C}=0.1$, $R_{d} / R_{b}=0.1$, and $E_{T h} / \Delta(0)=0.2$. (a) $s$-wave junctions. (b) $d$-wave junctions. (c) $p$-wave junctions.

$s$-wave junctions. The results at lower temperatures are shown in Fig. 2. The $I R$ product is enhanced with decreasing temperature because the proximity effect is enhanced. In this case the current-phase relation in $p$-wave junctions has a form close to $\sin (\varphi / 2)$, in contrast to the standard sinusoidal relation. This is a peculiar property of the formation of the resonant states in $p$-wave junctions where constructive interference occurs near $\varphi=\pi .^{43}$

Next we study the Josephson effect for other misorientational angles. As $\alpha$ or $\beta$ increase, the $I R$ product is monotonically suppressed due to the suppression of the proximity effect as shown in Figs. 3(a) and 3(b). In $d$ - (p)-wave junctions, the first harmonics disappear and hence the $I R$ product is proportional to $-\sin 2 \varphi$ at $(\alpha, \beta)=(\pi / 4,0)[(\pi / 2,0)]$ as shown in Figs. 3(c) and 3(d). This can be explained in the limiting case as follows. Near $T_{C}$, the $I R$ product, which stems from the first harmonics, is proportional to $\cos 2 \alpha \cos 2 \beta$ in $d$-wave junctions because angular averaging gives $\langle\cos (2 \phi-2 \alpha)\rangle \propto \cos 2 \alpha{ }^{44}$ Thus the first harmonics disappear at $\alpha=\pi / 4$. A similar argument is also applicable to $p$-wave junctions.

Let us discuss the results for the critical current. In Fig. 4, temperature dependence of the critical current is plotted for various $R_{d} / R_{b}$ and $E_{T h} / \Delta(0)$ in (a) $s$-wave, (b) $d$-wave, and (c) $p$-wave superconducting junctions with $Z=10$ and $(\alpha, \beta)=(0,0)$. As $R_{d} / R_{b}$ and $E_{T h} / \Delta(0)$ increase, $I_{C} R$ increases for all the junctions because the proximity effect is enhanced. In $p$-wave junctions, the critical current is strongly enhanced at low temperatures compared to the $s$-wave and $d$-wave junctions. When the misorientational angles in

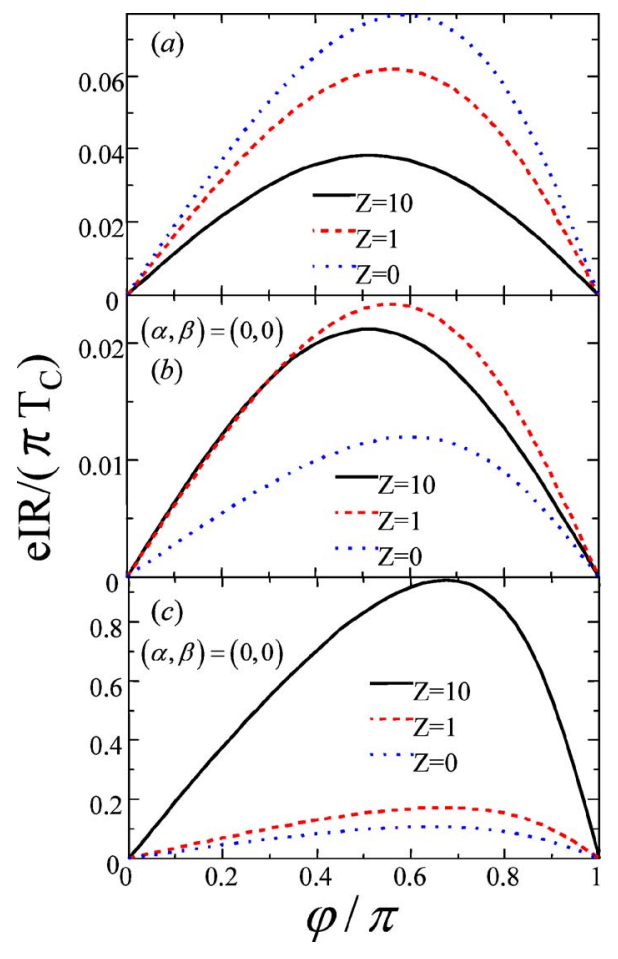

FIG. 2. (Color online) Current-phase relation for $T / T_{C}=0.02$, $R_{d} / R_{b}=0.1$, and $E_{T h} / \Delta(0)=0.2$. (a) $s$-wave junctions. (b) $d$-wave junctions. (c) $p$-wave junctions.

$d$-wave junctions are changed, nonmonotonic temperature dependence appears as shown in Fig. 5(a). This nonmonotonic behavior can be explained in terms of the competition between the proximity effect and the formation of MARSs. It is known from the previous studies that for $\alpha=\beta=0$ the proximity effect exists but the MARS is absent at the interfaces. On the other hand, for $\alpha=\beta=\pi / 4$, only the MARS exists and the proximity effect is absent. ${ }^{35,36}$ In other cases, both the proximity effect and MARSs are present. With the decrease of temperature, the formation of MARSs strongly suppresses the proximity effect. This results in the suppression of the Josephson current at low temperatures. Therefore, a nonmonotonic temperature dependence appears when both the proximity effect and MARSs coexist.

The above statement can be confirmed by calculation of the dependence of the anomalous Green's function $F$ on the Matsubara frequency $\omega$ as shown in Figs. 5(b) and 5(c) at $x=L / 2$ and $\varphi=\pi / 2$ for $(\alpha, \beta)=(\pi / 8,0)$. At low temperature $\left(T / T_{C}=0.01\right)$ the magnitude of $\operatorname{Im} F$ is suppressed at low energy in contrast to the case of high temperature $\left(T / T_{C}\right.$ $=0.2$ and 0.3 ). This result illustrates strong suppression of the proximity effect by the formation of MARSs at low $T$, which leads to the nonmonotonic temperature dependence. Note that this nonmonotonic dependence can appear only for large $Z$ when the role of MARSs is essential. ${ }^{40}$

It is interesting to study the junctions composed of superconductors with different symmetries. Here we study S/DN/D junctions with $Z=10, R_{d} / R_{b}=1$, and $E_{T h} / \Delta(0)=0.1$. We choose $T_{C D} / T_{C S}=5$ in Fig. 6(a) and $T_{C D} / T_{C S}=10$ in Fig. 6(b) where $T_{C S}\left(T_{C D}\right)$ denotes the critical temperature of the $s$-wave ( $d$-wave) superconductors. In this case the nonmono- 

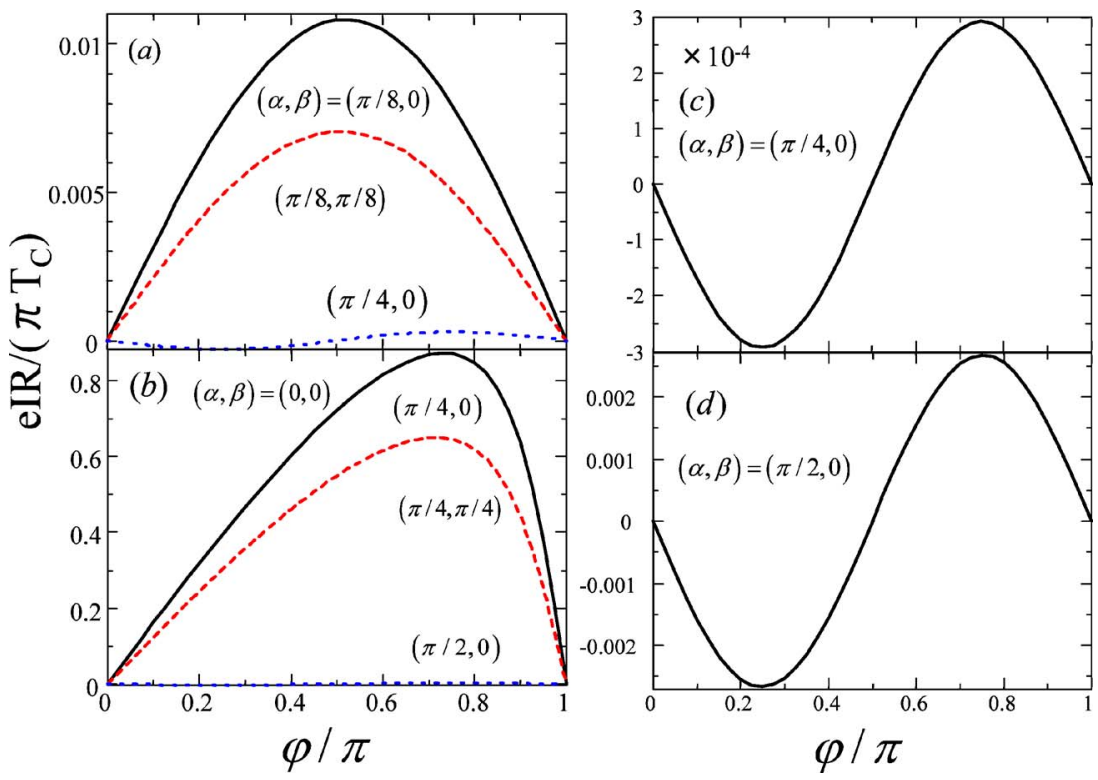

FIG. 3. (Color online) Current-phase relation for $T / T_{C}=0.01, \quad Z=10, \quad R_{d} / R_{b}=0.1, \quad$ and $E_{T h} / \Delta(0)=0.2$. (a) and (c) $d$-wave junctions. (b) and (d) $p$-wave junctions. tonic temperature dependence also occurs due to the competition as shown in Fig. 6. In S/insulator/D junctions, the nonmonotonic temperature dependence was observed experimentally in Ref. 45 . We can qualitatively explain these data by regarding the barrier as a diffusive normally conducting material.

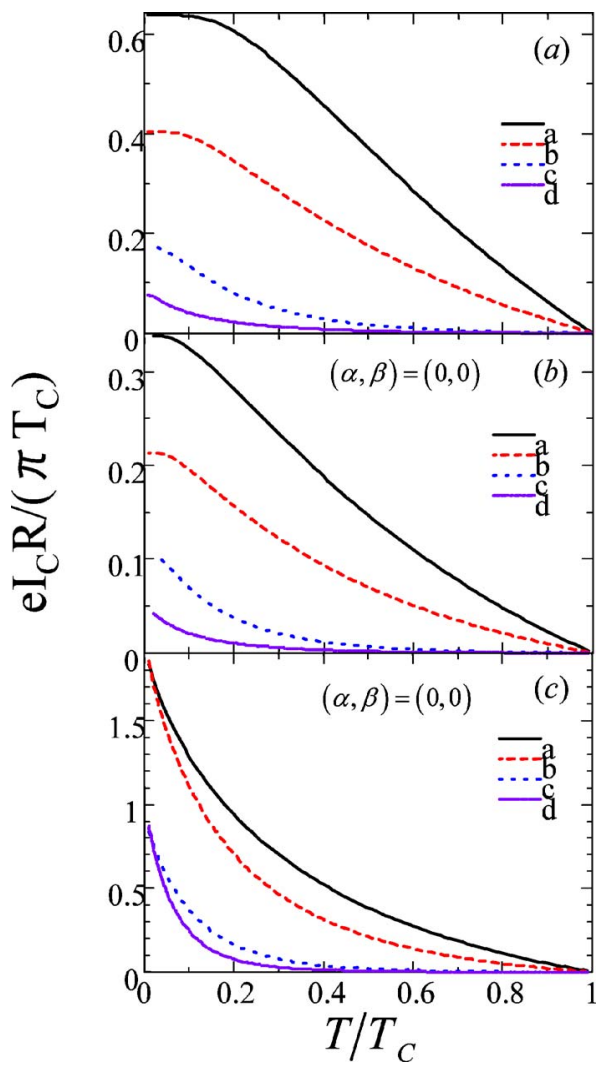

FIG. 4. (Color online) Temperature dependence of the critical current. (a) $s$-wave junctions. (b) $d$-wave junctions. (c) $p$-wave junctions. Curves $a . R_{d} / R_{b}=2$ and $E_{T h} / \Delta(0)=1$. Curves $b . R_{d} / R_{b}$ $=0.5$ and $E_{T h} / \Delta(0)=1$. Curves $c . R_{d} / R_{b}=2$ and $E_{T h} / \Delta(0)=0.1$. Curves $d . R_{d} / R_{b}=0.5$ and $E_{T h} / \Delta(0)=0.1$.
We study the dependence of the critical current on barrier thickness $L$ at various temperatures in (a) $s$-wave, (b) $d$-wave, and (c) $p$-wave superconducting junctions with $Z$ $=10, R_{d} / R_{b}=0.1$, and $(\alpha, \beta)=(0,0)$ in Fig. 7. The $I_{C} R$ prod-
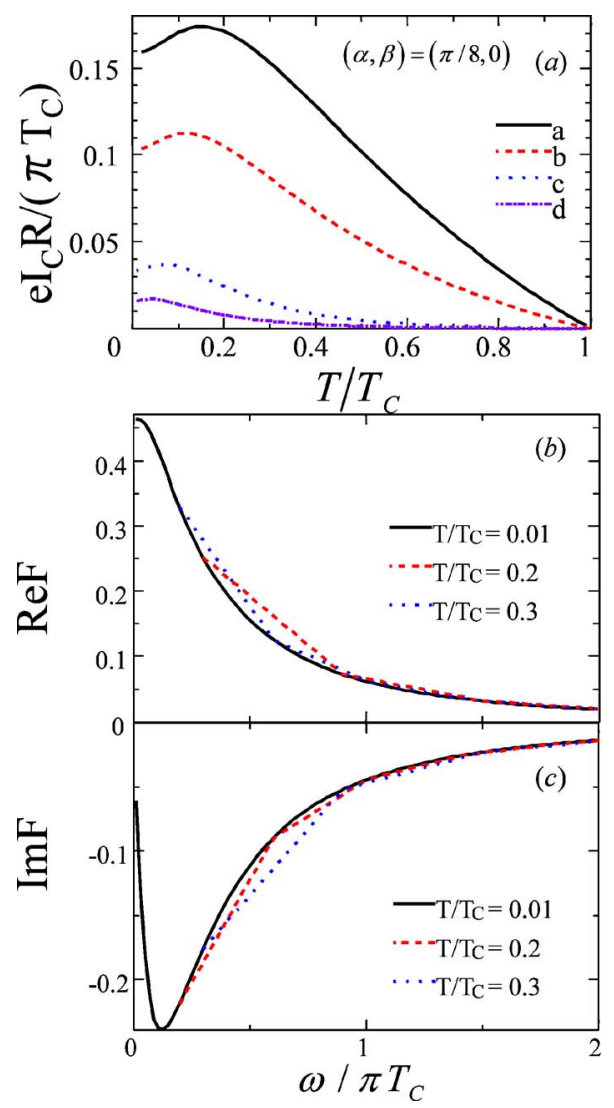

FIG. 5. (Color online) (a) Temperature dependence of the critical current for $(\alpha, \beta)=(\pi / 8,0)$. Curve $a . R_{d} / R_{b}=2$ and $E_{T h} / \Delta(0)$ $=1$. Curve $b . R_{d} / R_{b}=0.5$ and $E_{T h} / \Delta(0)=1$. Curve $c . R_{d} / R_{b}=2$ and $E_{T h} / \Delta(0)=0.1$. Curve $d . R_{d} / R_{b}=0.5$ and $E_{T h} / \Delta(0)=0.1$. (b) real and (c) imaginary parts of anomalous Green's functions $F$ with $R_{d} / R_{b}=2$ and $E_{T h} / \Delta(0)=1$. 


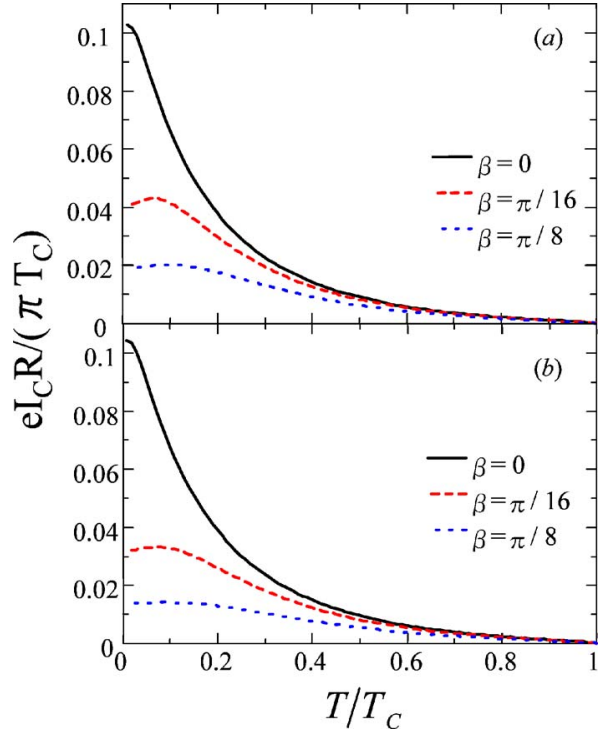

FIG. 6. (Color online) Temperature dependence of the critical current in S/DN/D junctions with $Z=10, R_{d} / R_{b}=1$, and $E_{T h} / \Delta(0)$ $=0.1 . T_{C D} / T_{C S}=$ (a) 5 and (b) 10 .

uct is proportional to $\exp (-\bar{C} L / \xi)$ for large $L / \xi$ for all the junctions as shown in Fig. 7. Here $\bar{C}$ is a constant independent of $L$. As temperature is lowered, the magnitude of $\bar{C}$ is reduced. From our results, we also find the relation $\bar{C}$ $\propto T^{-1 / 2}$. The results for junctions with other misorientational angles for $T / T_{C}=0.01, Z=10$, and $R_{d} / R_{b}=0.1$ are shown in

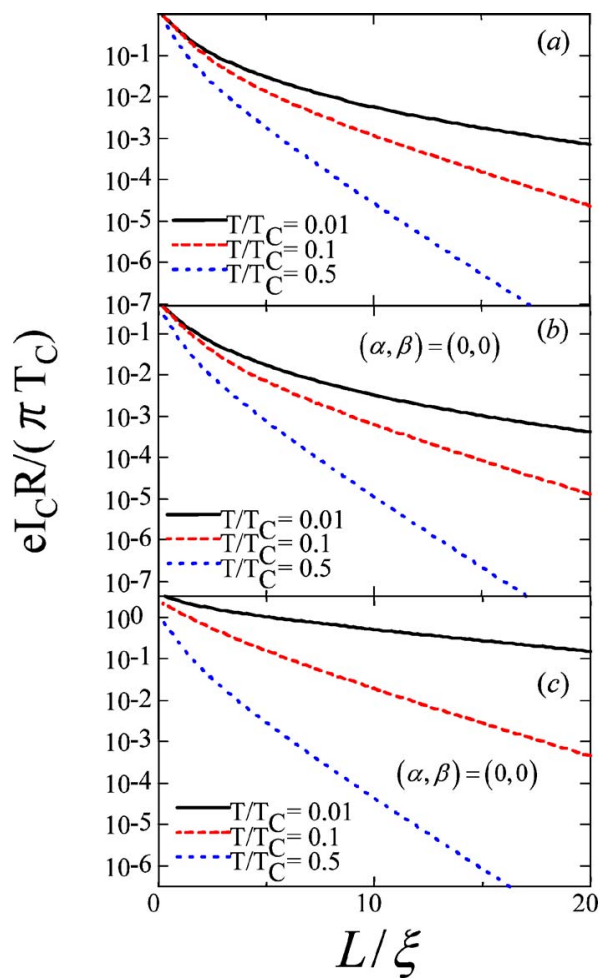

FIG. 7. (Color online) Length dependence of the critical current with $Z=10$ and $R_{d} / R_{b}=0.1$. (a) $s$-wave junctions. (b) $d$-wave junctions. (c) $p$-wave junctions.

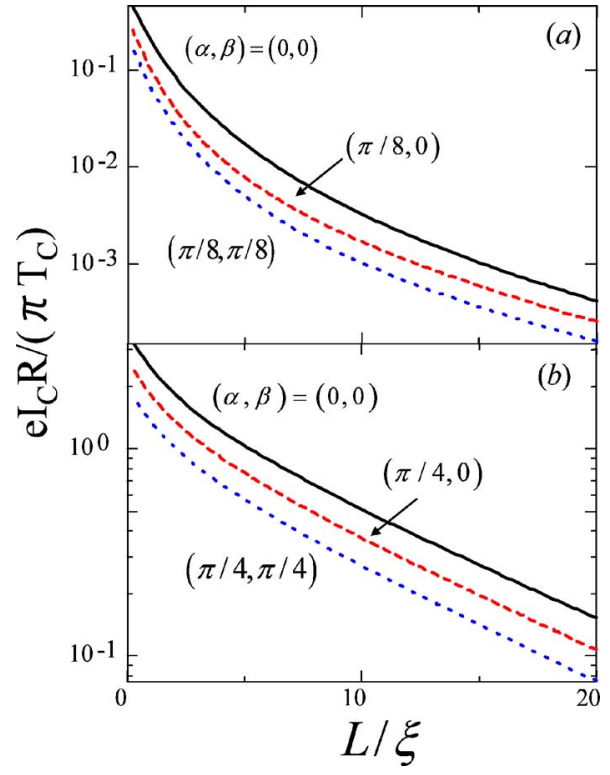

FIG. 8. (Color online) Length dependence of the critical current with $T / T_{C}=0.01, Z=10$, and $R_{d} / R_{b}=0.1$. (a) $d$-wave junctions. (b) $p$-wave junctions.

Fig. 8. As $\alpha$ and $\beta$ increase, $I_{C} R$ is suppressed. However, $\bar{C}$ is independent of these values, which indicates that MARSs do not influence the effective coherence length $\xi / \bar{C}$. This is because the effective coherence length reflecting the penetration of Cooper pairs is determined by the Usadel equation and therefore is independent of MARSs.

\section{B. Junctions with DFs}

Here we consider junctions with DFs. We will study three types of junctions: D/DF/D, P/DF/P and S/DF/P junctions. Figure 9 shows the current-phase relation in $\mathrm{D} / \mathrm{DF} / \mathrm{D}$ junc-

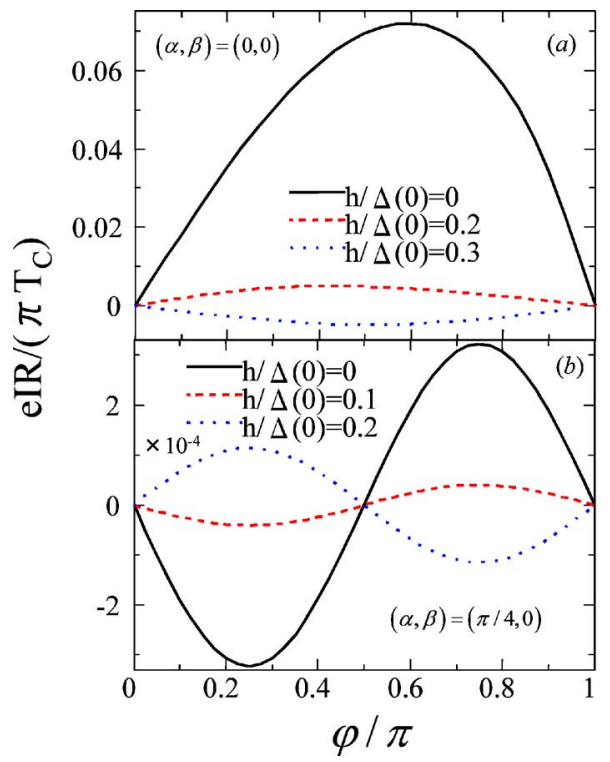

FIG. 9. (Color online) Current-phase relation in D/DF/D junctions for $T / T_{C}=0.01, Z=10, R_{d} / R_{b}=1$, and $E_{T h} / \Delta(0)=0.1$. 


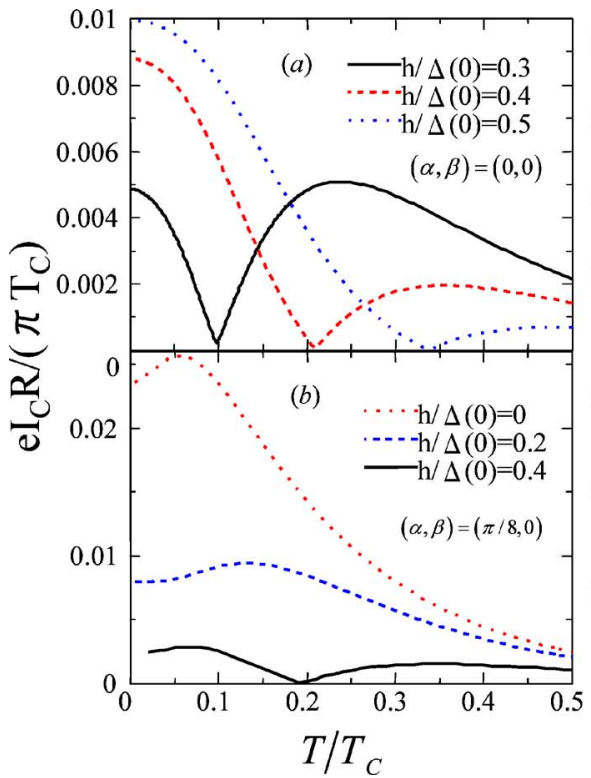

FIG. 10. (Color online) Temperature dependence of the critical current in D/DF/D junctions. $Z=10$ in (a) and (b). $h / \Delta(0)=0.4$ in (c) and (d). tions for $T / T_{C}=0.01, Z=10, R_{d} / R_{b}=1$, and $E_{T h} / \Delta(0)=0.1$. At $(\alpha, \beta)=(0,0)$ where the MARSs are absent, the exchange field causes a $0-\pi$ transition as predicted for $s$-wave junctions [see Fig. 9(a)]. Similarly, the second harmonic changes its sign at $(\alpha, \beta)=(\pi / 4,0)$, where the proximity effect is absent at $x=0$, as shown in Fig. 9(b). Figure 10 displays the temperature dependence of the critical current in D/DF/D junctions with $R_{d} / R_{b}=1$ and $E_{T h} / \Delta(0)=0.1$. At $(\alpha, \beta)$ $=(0,0)$, the exchange field causes a $0-\pi$ transition as shown in Fig. 10(a). At $(\alpha, \beta)=(\pi / 8,0)$, the exchange field also causes a $0-\pi$ transition, and, as a result, a double-peak structure appears for $h / \Delta(0)=0.4$ as shown in Fig. 10(b). The peak at lower temperature stems from the competition between proximity effect and MARSs. The peak at higher temperature stems from the $0-\pi$ transition. With the decrease of $Z$, the magnitude of $I_{C} R$ is suppressed while the $0-\pi$ transition temperature is almost independent of $Z$ [see Fig. 10(c)]. At $(\alpha, \beta)=(\pi / 8,0)$, the peak at lower temperature disappears for small $Z$ as shown in Fig. 10(d) because the existence of the insulating barrier is essential for the formation of MARSs.

The barrier thickness dependence of the critical current in D/DF/D junctions is plotted in Fig. 11 with $T / T_{C}=0.1, Z$ $=10, R_{d} / R_{b}=1$, and $E_{T h} / \Delta(0)=0.1$. For $h=0$, the $I_{C} R$ product has an exponential dependence on $L$. As $h / \Delta(0)$ increases, $I_{C}$ oscillates as a function of $L / \xi$. The period of the oscillation becomes shorter with increasing $h$ as shown in Fig. 11(a). As $\alpha$ and $\beta$ increase, $I_{C} R$ is suppressed while the period of the oscillations remains constant, that is, the period is independent of the MARSs. The second harmonics have a shorter (almost half) oscillation period than that of the first harmonics, similar to the predictions for S/DF/S junctions ${ }^{14,15}$ [see the result for $(\alpha, \beta)=(\pi / 4,0)$ in Fig. $11(\mathrm{~b})]$.

Next we consider the P/DF/P junctions. The current-phase relation in $\mathrm{P} / \mathrm{DF} / \mathrm{P}$ junctions for $T / T_{C}=0.01, Z=10, R_{d} / R_{b}$ $=1$, and $E_{T h} / \Delta(0)=0.1$ is plotted in Fig. 12. With increasing $h$, the dependence of $I R$ changes from $\sin (\varphi / 2)$ to $\sin 2 \varphi$ and finally to $-\sin \varphi$ at $(\alpha, \beta)=(0,0)$ as shown in Fig. 12(a). The phase dependences originate from the formation of the resonant states, the disappearance of the first harmonics at the $0-\pi$ transition and the emergence of the $\pi$ junctions, respectively. At $(\alpha, \beta)=(\pi / 2,0)$ where the MARSs are absent at $x=0$, the second harmonics change the sign with the increase of $h$ as shown in Fig. 12(b). The critical current as a function of $T$ is shown in Fig. 13(a). The $0-\pi$ transition occurs due to the exchange field. Similarly, as $h / \Delta(0)$ increases, $I_{C} R$ oscillates as a function of $L / \xi$. The period of the oscillation becomes short with increasing $h$ as shown in Fig. 13(b).

Finally we study S/DF/P junctions for $Z=10, R_{d} / R_{b}=1$, $E_{T h} / \Delta(0)=0.1$, and $\beta=0$. The current-phase relation at $T / T_{C}=0.01$ has the form of $-\sin 2 \varphi$ for $h=0$ due to the difference of the parities of two superconductors ${ }^{41}$ as shown in Fig. 14(a). As $h$ increases, the shape of $I R$ transforms from

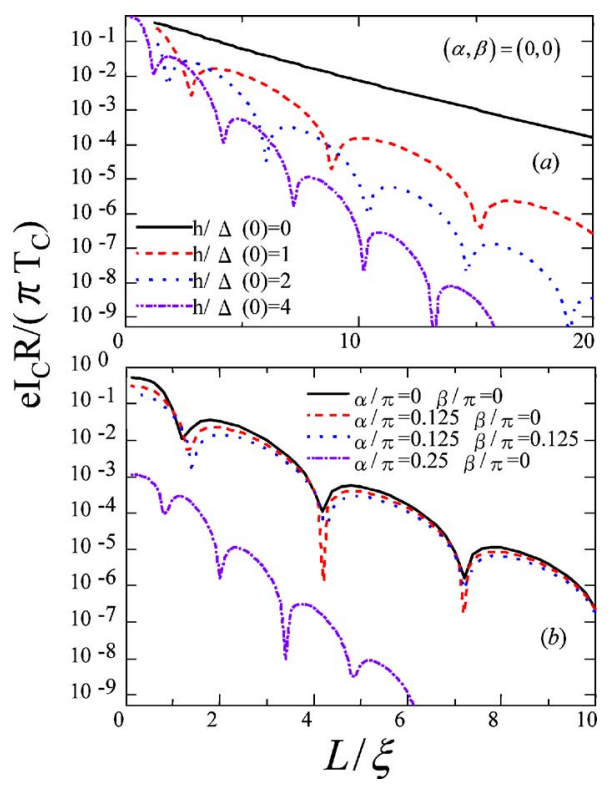

FIG. 11. (Color online) Length dependence of the critical current in $\mathrm{D} / \mathrm{DF} / \mathrm{D}$ junctions with $T / T_{C}=0.1, Z=10, R_{d} / R_{b}=1$, and $E_{T h} / \Delta(0)=0.1$. We choose $h / \Delta(0)=4$ in (b). 


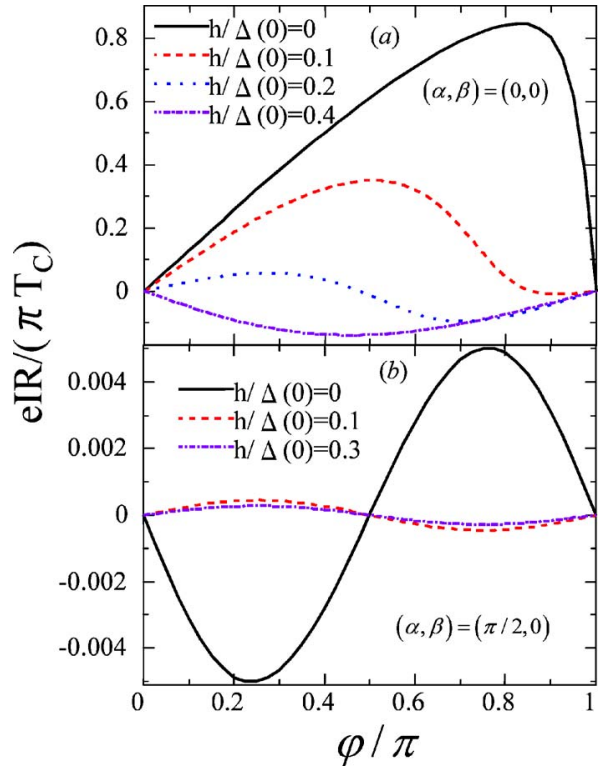

FIG. 12. (Color online) Current-phase relation in P/DF/P junctions for $T / T_{C}=0.01, Z=10, R_{d} / R_{b}=1$, and $E_{T h} / \Delta(0)=0.1$.

$-\sin 2 \varphi$ to $\cos \varphi$ since the first harmonics recover by breaking the symmetry between up and down spins. The temperature dependence of the critical current is plotted in Fig. 14(b). The magnitude of $I_{C} R$ is enhanced by the increase of $h$ due to the recovery of the first harmonics, in contrast to junctions between superconductors with equal parities.

\section{CONCLUSIONS}

In this paper, we studied the Josephson effect in junctions between unconventional superconductors with diffusive bar-

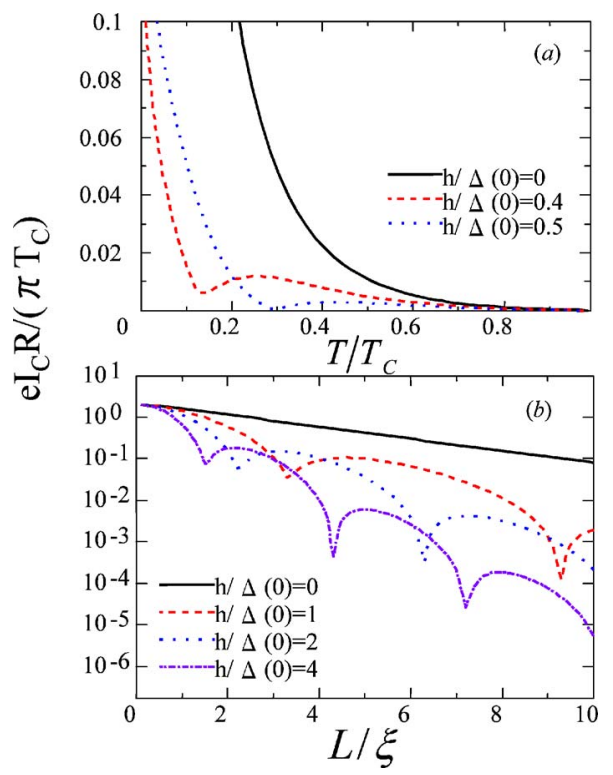

FIG. 13. (Color online) (a) Temperature and (b) length dependence of the critical current in $\mathrm{P} / \mathrm{DF} / \mathrm{P}$ junctions. $Z=10, R_{d} / R_{b}=1$, and $(\alpha, \beta)=(0,0)$. We choose $E_{T h} / \Delta(0)=0.1$ in (a) and $T / T_{C}=0.1$ in (b).
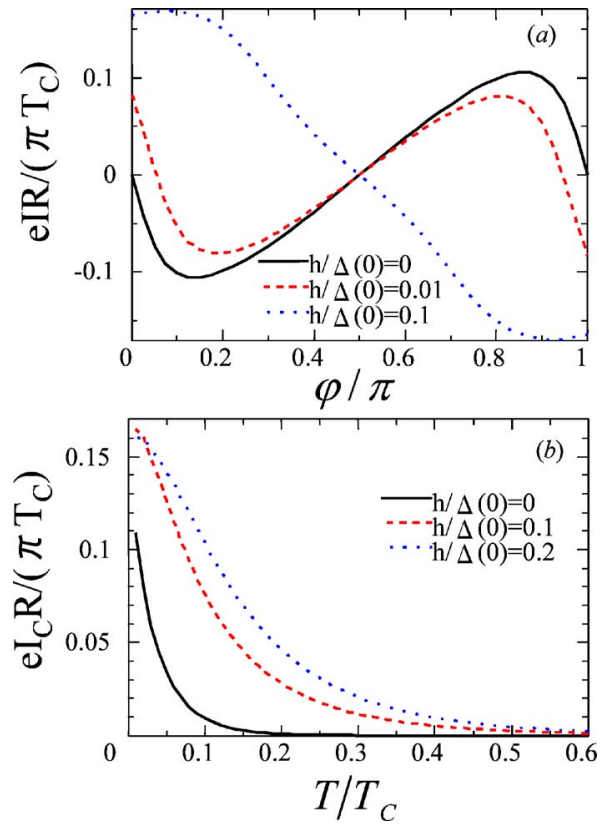

FIG. 14. (Color online) (a) Current-phase relation at $T / T_{C}$ $=0.01$ and $(\mathrm{b})$ temperature dependence of the critical current in $\mathrm{S} / \mathrm{DF} / \mathrm{P}$ junctions for $\mathrm{Z}=10, R_{d} / R_{b}=1, E_{T h} / \Delta(0)=0.1$, and $\beta=0$.

riers. The Usadel equations in the barrier region were solved with the generalized boundary conditions applicable to unconventional superconductors at the interfaces. Applying these boundary conditions, we calculated the Josephson current in various types of junctions: S/DN/S, D/DN/D, P/DN/P, S/DN/D, D/DF/D, P/DF/P, and S/DF/P junctions. Our main conclusions can be summarized as follows.

(1) The dependences of Josephson current on the interface barrier strength $Z$ are different for S/DN/S, D/DN/D, and $\mathrm{P} / \mathrm{DN} / \mathrm{P}$ junctions. The Josephson current is suppressed by the increase of $Z$ in $S / D N / S$ junctions while it is enhanced by the increase of $Z$ in $\mathrm{D} / \mathrm{DN} / \mathrm{D}$ and $\mathrm{P} / \mathrm{DN} / \mathrm{P}$ junctions. In $\mathrm{D} / \mathrm{DN} / \mathrm{D}$ and $\mathrm{P} / \mathrm{DN} / \mathrm{P}$ junctions, the proximity effect is enhanced by the increase of $Z$ due to the cancellation of the positive and negative parts of the pair potential in the angular averaging and the coexistence of MARSs and proximity effect, respectively. The coexistence also induces anomalous current-phase relation in P/DN/P junctions. When the proximity effect is absent at one interface, the second harmonics dominate in $\mathrm{D} / \mathrm{DN} / \mathrm{D}$ and $\mathrm{P} / \mathrm{DN} / \mathrm{P}$ junctions. The competition between MARSs and proximity effect causes a nonmonotonic temperature dependence of the critical current in D/DN/D junctions. Similar dependence can be seen in S/DN/D junctions.

(2) In S/DN/S, D/DN/D, and P/DN/P junctions, the critical current has an exponential dependence on the length of the DN. The prefactor of the length is independent of the MARSs.

(3) In D/DF/D, P/DF/P, and S/DF/P junctions, the $\pi$ state can be realized. A double-peak structure in temperature dependence of the critical current occurs in D/DF/D junctions due to $0-\pi$ transition and the competition between MARSs and proximity effect. In S/DF/P junctions, the Josephson current can be enhanced by the exchange field, in contrast to 
other types of junctions, due to the recovery of the first harmonics.

(4) In $\mathrm{D} / \mathrm{DF} / \mathrm{D}$ and $\mathrm{P} / \mathrm{DF} / \mathrm{P}$ junctions, the critical current has an oscillatory behavior as a function of the length of the DF. The period of the oscillation becomes short for large exchange fields while it is independent of the MARSs. The second harmonics show almost half periodicity compared to the first harmonics.

\section{ACKNOWLEDGMENTS}

The authors thank T. Akazaki of NTT Basic Research Laboratories for useful discussions. T.Y. acknowledges support by JSPS. This work was supported by the NTT basic research laboratory, NAREGI Nanoscience Project, the Min- istry of Education, Culture, Sports, Science and Technology, Japan, the Core Research for Evolutional Science and Technology (CREST) of the Japan Science and Technology Corporation (JST), a Grant-in-Aid for Scientific Research on Priority Area "Novel Quantum Phenomena Specific to Anisotropic Superconductivity" (Grant No. 17071007) from the Ministry of Education, Culture, Sports, Science and Technology of Japan, a Grant-in-Aid for Scientific Research on B (Grant No. 17340106) from the Ministry of Education, Culture, Sports, Science and Technology of Japan, and the 21st Century COE "Frontiers of Computational Science." The computational aspect of this work was performed at the Research Center for Computational Science, Okazaki National Research Institutes, and the facilities of the Supercomputer Center, Institute for Solid State Physics, University of Tokyo and the Computer Center.
${ }^{1}$ B. D. Josephson, Phys. Lett. 1, 251 (1962).

${ }^{2}$ P. G. de Gennes, Rev. Mod. Phys. 36, 225 (1964).

${ }^{3}$ K. K. Likharev, Rev. Mod. Phys. 51, 101 (1979).

${ }^{4}$ A. A. Golubov, M. Yu. Kupriyanov, and E. Il'ichev Rev. Mod. Phys. 76, 411 (2004).

${ }^{5}$ M. Yu. Kupriyanov and V. F. Lukichev, Sov. Phys. JETP 67, 1163 (1988).

${ }^{6}$ A. V. Zaitsev, Physica C 185-189, 2539 (1991).

${ }^{7}$ A. I. Buzdin, Rev. Mod. Phys. 77, 935 (2005).

${ }^{8}$ F. S. Bergeret, A. F. Volkov, and K. B. Efetov, Rev. Mod. Phys. 77, 1321 (2005).

${ }^{9}$ L. N. Bulaevskii, V. V. Kuzii, and A. A. Sobyanin, JETP Lett. 25, 290 (1977).

${ }^{10}$ A. I. Buzdin, L. N. Bulaevskii, and S. V. Panjukov, JETP Lett. 35, 178 (1982).

${ }^{11}$ A. I. Buzdin, B. Bujicic, and B. M. Yu. Kupriyanov, Sov. Phys. JETP 74, 124 (1992).

${ }^{12}$ E. A. Demler, G. B. Arnold, and M. R. Beasley, Phys. Rev. B 55, 15174 (1997).

${ }^{13}$ A. A. Golubov, M. Yu. Kupriyanov, and Ya. V. Fominov, JETP Lett. 75, 223 (2002).

${ }^{14}$ A. Buzdin, Phys. Rev. B 72, 100501(R) (2005).

${ }^{15}$ M. Houzet, V. Vinokur, and F. Pistolesi, Phys. Rev. B 72, 220506(R) (2005).

${ }^{16}$ M. Faure, A. I. Buzdin, A. A. Golubov, and M. Yu. Kupriyanov, Phys. Rev. B 73, 064505 (2006).

${ }^{17}$ P. Fulde and R. A. Ferrel, Phys. Rev. 135, A550 (1964).

${ }^{18}$ A. I. Larkin and Yu. N. Ovchinnikov, Zh. Eksp. Teor. Fiz. 47, 1136 (1964) [Sov. Phys. JETP 20, 762 (1965)].

${ }^{19}$ V. V. Ryazanov, V. A. Oboznov, A. Yu. Rusanov, A. V. Veretennikov, A. A. Golubov, and J. Aarts, Phys. Rev. Lett. 86, 2427 (2001).

${ }^{20}$ T. Kontos, M. Aprili, J. Lesueur, F. Genet, B. Stephanidis, and R. Boursier Phys. Rev. Lett. 89, 137007 (2002).

${ }^{21}$ H. Sellier, C. Baraduc, F. Lefloch, and R. Calemczuk, Phys. Rev. Lett. 92, 257005 (2004).

${ }^{22}$ S. M. Frolov, D. J. Van Harlingen, V. A. Oboznov, V. V. Bolginov, and V. V. Ryazanov, Phys. Rev. B 70, 144505 (2004).

${ }^{23}$ Y. Blum, A. Tsukernik, M. Karpovski, and A. Palevski, Phys.
Rev. B 70, 214501 (2004)

${ }^{24}$ C. Surgers et al., J. Magn. Magn. Mater. 240, 598 (2002).

${ }^{25}$ C. Bell, R. Loloee, G. Burnell, and M. G. Blamire, Phys. Rev. B 71, 180501(R) (2005); J. W. A. Robinson, S. Piano, G. Burnell, C. Bell, and M. G. Blamire, Phys. Rev. Lett. 97, 177003 (2006).

${ }^{26}$ V. Shelukhin, A. Tsukernik, M. Karpovski, Y. Blum, K. B. Efetov, A. F. Volkov, T. Champel, M. Eschrig, T. Lofwander, G. Schon, and A. Palevski, Phys. Rev. B 73, 174506 (2006).

${ }^{27}$ M. Weides, K. Tillmann, and H. Kohlstedt, Physica C 437-438, 349 (2006); M. Weides, M. Kemmler, H. Kohlstedt, A. Buzdin, E. Goldobin, D. Koelle, and R. Kleiner, Appl. Phys. Lett. 89, 122511 (2006).

${ }^{28}$ G. P. Pepe, R. Latempa, L. Parlato, A. Ruotolo, G. Ausanio, G. Peluso, A. Barone, A. A. Golubov, Ya. V. Fominov, and M. Yu. Kupriyanov, Phys. Rev. B 73, 054506 (2006).

${ }^{29}$ F. Born, M. Siegel, E. K. Hollmann, H. Braak, A. A. Golubov, D. Yu. Gusakova, and M. Yu. Kupriyanov, Phys. Rev. B 74, 140501(R) (2006).

${ }^{30}$ L. J. Buchholtz and G. Zwicknagl, Phys. Rev. B 23, 5788 (1981); C. Bruder, ibid. 41, 4017 (1990); C. R. Hu, Phys. Rev. Lett. 72, 1526 (1994).

${ }^{31}$ Y. Tanaka and S. Kashiwaya, Phys. Rev. Lett. 74, 3451 (1995); S. Kashiwaya, Y. Tanaka, M. Koyanagi, and K. Kajimura, Phys. Rev. B 53, 2667 (1996); S. Kashiwaya and Y. Tanaka, Rep. Prog. Phys. 63, 1641 (2000).

${ }^{32}$ Yu. S. Barash, H. Burkhardt, and D. Rainer, Phys. Rev. Lett. 77, 4070 (1996); Y. Tanaka and S. Kashiwaya, Phys. Rev. B 56, 892 (1997).

${ }^{33}$ A. A. Golubov and M. Yu. Kupriyanov, JETP Lett. 69, 262 (1999).

${ }^{34}$ Yu. V. Nazarov, Superlattices Microstruct. 25, 1221 (1999).

${ }^{35}$ Y. Tanaka, Y. V. Nazarov, and S. Kashiwaya, Phys. Rev. Lett. 90, 167003 (2003).

${ }^{36}$ Y. Tanaka, Yu. V. Nazarov, A. A. Golubov, and S. Kashiwaya, Phys. Rev. B 69, 144519 (2004).

${ }^{37}$ Y. Tanaka and S. Kashiwaya, Phys. Rev. B 70, 012507 (2004); Y. Tanaka, S. Kashiwaya, and T. Yokoyama, ibid. 71, 094513 (2005).

${ }^{38}$ Y. Asano, Phys. Rev. B 63, 052512 (2001); 64, 014511 (2001); 
64, 224515 (2001); J. Phys. Soc. Jpn. 71, 905 (2002).

${ }^{39}$ K. D. Usadel, Phys. Rev. Lett. 25, 507 (1970).

${ }^{40}$ T. Yokoyama, Y. Tanaka, A. A. Golubov, and Y. Asano, Phys. Rev. B 73, 140504(R) (2006).

${ }^{41}$ Y. Tanaka and S. Kashiwaya, J. Phys. Soc. Jpn. 68, 3485 (1999).

${ }^{42}$ T. Yokoyama, Y. Tanaka, and A. A. Golubov, Phys. Rev. B 72, 052512 (2005); 73, 094501 (2006); T. Yokoyama and Y. Tanaka,
C. R. Phys. 7, 136 (2006).

${ }^{43}$ Y. Asano, Y. Tanaka, and S. Kashiwaya, Phys. Rev. Lett. 96, 097007 (2006); Y. Asano, Y. Tanaka, T. Yokoyama, and S. Kashiwaya, Phys. Rev. B 74, 064507 (2006).

${ }^{44}$ T. Yokoyama, Y. Sawa, Y. Tanaka, and A. A. Golubov, Phys. Rev. B 75, 020502(R) (2007).

${ }^{45}$ I. Iguchi and Z. Wen, Phys. Rev. B 49, R12388 (1994). 